\title{
Monitoramento de metais nos lagos igapó em Londrina, PR, usando a metodologia de EDXRF
}

\section{Metal monitoring in igapó lakes in Londrina, Paraná, Brazil, using EDXRF methodology}

\author{
Fábio L. Melquiades ${ }^{1}$; Gelsy W. P. Soares Gonçalves²; Wislley D. Silva ${ }^{3}$; \\ Paulo S. Parreira ${ }^{4}$; Carlos R. Appoloni ${ }^{5}$
}

\section{Resumo}

O objetivo deste trabalho é apresentar os resultados de monitoramento de metais nos Lagos Igapó, na cidade de Londrina, e avaliar o potencial de um sistema portátil de EDXRF para determinação da concentração de metais totais em água, como uma metodologia analítica alternativa, comparada com AAS. Considerando todos os pontos de amostragem, foi possível quantificar por EDXRF a seguinte faixa de concentrações, em $\mathrm{mg} \mathrm{L}^{-1}$, para $\mathrm{Ca}(0,043-0,021)$, Ti $(0,137-0,014), \mathrm{Mn}(0,052-0,008), \mathrm{Fe}$ $(1,046-0,035), \mathrm{Ni}(0,012-0,010), \mathrm{Cu}(0,010-0,007)$ and $\mathrm{Zn}(0,070-0,007)$. Os resultados de AAS e EDXRF forma complementares, gerando conclusões relevantes sobre a análise.

Palavras-chave: Metal. Água. Fluorescência de Raios X.

\begin{abstract}
The purpose of this work is to present the results of the metal monitoring in Igapó Lakes, in the city of Londrina, as well as to evaluate the potential of a portable EDXRF system for the determination of total metal concentration in water, as an alternative analytical methodology, compared to AAS. Considering all the sampling points, it was possible to quantify by EDXRF, the following range of concentrations, in $\mathrm{mg} \mathrm{L}^{-1}$, for $\mathrm{Ca}(0.043-0.021)$, $\mathrm{Ti}(0.137-0.014), \mathrm{Mn}(0.052-0.008), \mathrm{Fe}(1.046-0.035), \mathrm{Ni}(0.012$ $-0.010), \mathrm{Cu}(0.010-0.007)$ and $\mathrm{Zn}(0.070-0.007)$. AAS and EDXRF results were complementary, generating relevant conclusions about the analysis.
\end{abstract}

Key words: Metal. Water. X-ray fluorescence.

1 Docente do Departamento de Física, Universidade Estadual do Centro-Oeste; E-mail: fmelquiades@unicentro.br

Instituto Ambiental do Paraná, Secretaria Regional de Londrina .

Graduando do Curso de Física, Universidade Estadual de Londrina.

4 Físico do Laboratório de Física Nuclear Aplicada, Departamento de Física, Universidade Estadual de Londrina.

5 Docente do Departamento de Física, Universidade Estadual de Londrina; E-mail: appoloni@uel.br 


\section{Introdução}

A água é um dos constituintes mais importantes do meio ambiente, sendo imprescindível para vida humana. A preservação de fontes de água doce tornou-se uma das prioridades ambientais no mundo. O controle de qualidade é particularmente importante no caso de água potável, Esão necessárias técnicas analíticas altamente sensíveis para medir pequenas concentrações de elementos essenciais e tóxicos. A contaminação surge principalmente da descarga de resíduos de atividades humanas e naturais, que resultam em uma interferência indesejável no uso da água (MELQUIADES, APPOLONI, 2004).

A determinação, com acurácia, da concentração total de traços de metais em matrizes ambientais, como material particulado do ar residencial e urbano, poeira, solos, água e sedimentos, representa um desafio significativo. Isso tem conduzido ao desenvolvimento e ao aperfeiçoamento contínuo de vários métodos analíticos de digestão e préconcentração de amostras, e ao desenvolvimento de técnicas instrumentais para obter diversas informações químicas em um período de tempo relativamente curto (HASSAN et al., 2007).

A determinação de elementos pesados e tóxicos permite o estudo de sua distribuição, o nível de poluição, bem como o risco oferecido pelo ecossistema estudado. Sem dúvida, as metodologias mais utilizadas para estes propósitos atualmente são a Espectrometria de Absorção Atômica (AAS), a Espectrometria de Emissão Atômica com uso de plasma (ICP-AES) e a Análise por Ativação Neutrônica (NAA). A necessidade de um reator nuclear para NAA, a da preparação exaustiva de amostra para AAS e ICP-AES, quando comparada à Fluorescência de Raios x por Dispersão em Energia (EDXRF), estimula o interesse em direção à EDXRF em pesquisas ambientais (HOU, JONES, 2000). A EDXRF é uma técnica não destrutiva e multi-elementar, que pode ser aplicada a qualquer tipo de amostra, liquida, sólida, espessa ou fina, em muitos casos com sensibilidade suficiente para quantificação de diversos elementos (CESAREO, CASTELLANO; CUEVAS, 1998)

A técnica de XRF é um método bem estabelecido na área de pesquisas ambientais, sendo uma das técnicas de espectrometria atômica que podem ser adaptadas para uso em campo com equipamentos portáteis (IVANOVA, DJINGOVA, KULLEF, 1998). É uma técnica analítica multi-elementar, rápida, de baixo custo, de fácil operação e seus princípios físicos, vantagens e limitações são bem conhecidos(BERTIN, 1975, VAN GRIEKEN, 1990, CESAREO, 2000).

O objetivo deste trabalho é apresentar os resultados de monitoramento de metais nos Lagos Igapó, na cidade de Londrina. Os lagos Igapó cruzam a região central da cidade e fazem parte da microbacia do Ribeirão Cambé, que abastece os lagos. Eles são utilizados pela população para recreação, pesca e esportes aquáticos. O Instituto Ambiental do Paraná (IAP), que é a agência responsável por fiscalizar a região, realiza coletas de água duas vezes por ano nos lagos. As análises para quantificação de metais são feitas por AAS.

Além do monitoramento, outro objetivo foi avaliar o potencial de um sistema portátil de EDXRF como uma metodologia analítica alternativa para determinação da concentração de metais totais em água, quando comparada com AAS. Assim, as amostras foram analisadas por ambas as técnicas.

\section{Experimental}

\section{Local de amostragem}

Foram monitorados 10 pontos de amostragem do Lago Igapó e seus afluentes, como mostra a Figura 1.

\section{Preparação das amostras}

Realizaram-se três coletas no lago Igapó e seus afluentes, no verão e inverno de 2006 e no inverno de 2007. 
Foram coletados dois litros de água superficial em garrafas de polietielno. Um litro foi utilizado para análise por AAS e outro por EDXRF.

As amostras foram levadas para laboratório e preparadas no mesmo dia para as análises por EDXRF. Primeiramente elas foram filtradas, em membranas de ésteres de celulose de $0,45 \mu \mathrm{m}$ de tamanho de poro, para retenção do material particulado em suspensão (MPS).

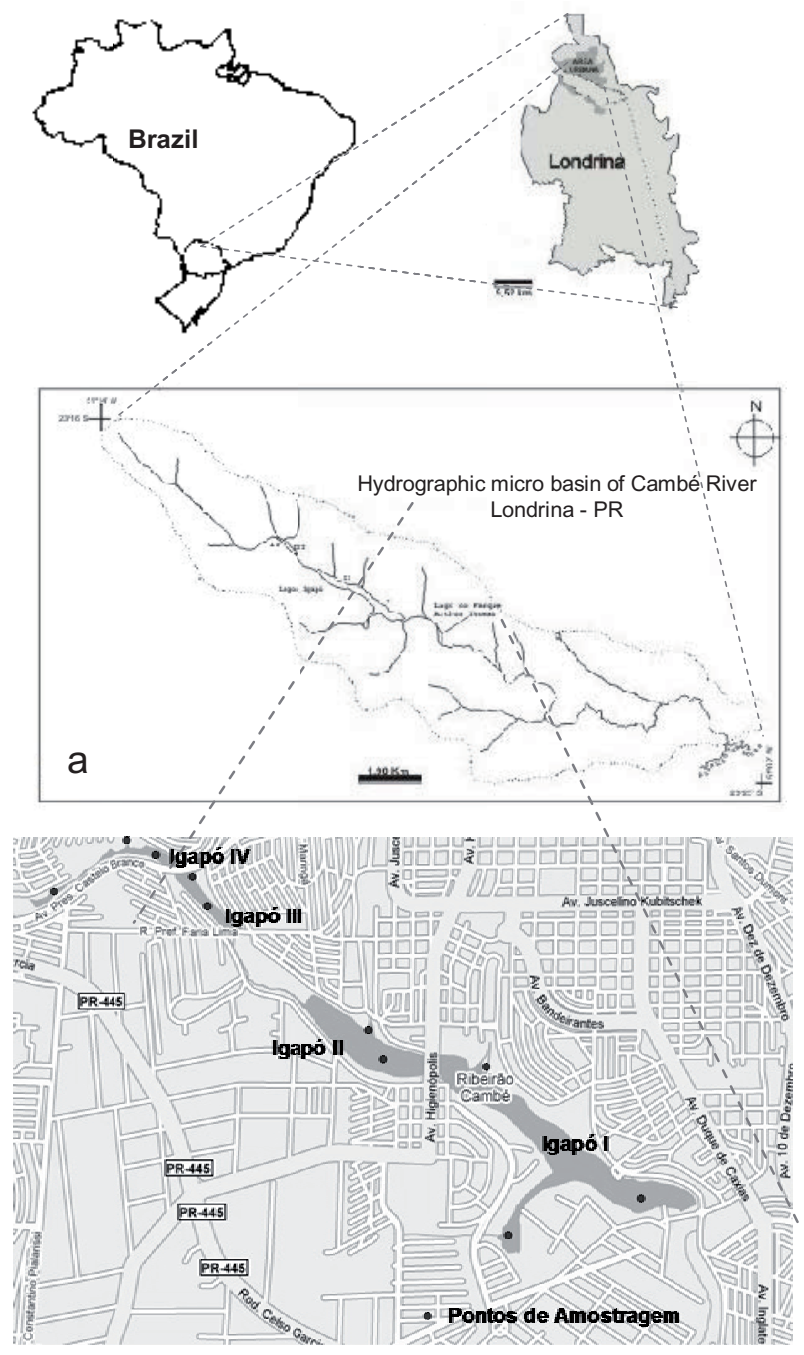

Figura 1. (a) Identificação da região de análise. (b) Pontos de coleta de amostras nos Lagos Igapó e seus afluentes

Para atingir os níveis de concentração máximos exigidos nacional e internacionalmente pelas agencias fiscalizadoras, uma etapa de pré- concentração é essencial. A metodologia usada foi a precipitação com agente quelante Ammonium Pyrrolydine Dithiocarbamate (APDC). Assim, as amostras percoladas foram submetidas ao seguinte procedimento de pré-concentração: $300 \mathrm{~mL}$ de solução são ajustados para $\mathrm{pH}$ 4, em seguida, $1 \mathrm{~mL}$ de APDC 2\% é adicionado a solução e agitado por 10 minutos e depois filtrados em membranas de ésteres de celulose de $0.45 \mu \mathrm{m}$ (MELQUIADES et al., 2007). As membranas são secas à temperatura ambiente, por $24 \mathrm{~h}$. Foram preparadas 3 membranas de cada amostra utilizando o procedimento de préconcentração.

Das membranas com MPS é obtida a fração de metais não dissolvida e das membranas préconcentradas, a fração dissolvida. Todas as membranas foram irradiadas 3 vezes pela técnica de EDXRF.

Para a determinação por AAS, as amostras foram preservadas com $\mathrm{HNO}_{3}$ até a análise. A preparação consiste na digestão de $100 \mathrm{~mL}$ de amostra com 5 $\mathrm{mL}$ de $\mathrm{HNO}_{3}$ até a evaporação total. Então, mais 5 $\mathrm{mL}$ de $\mathrm{HNO}_{3}$ são adicionados, a solução é aquecida e $1 \mathrm{~mL}$ da mesma é diluído em água destilada para $25 \mathrm{~mL}$.

\section{Instrumentação de EDXRF}

O sistema de medidas é composto por um detector de Si-PIN (221 eV de resolução para a linha de $5.9 \mathrm{keV}$ ) (AMPTEK Inc.), acoplado a um pré-amplificador, uma fonte de alta tensão com amplificador, um analisador multicanal e a um notebook para aquisição e análise dos dados. A excitação das amostras foi realizada com um mini tubo de raios X (alvo de Ag, 4W) da MOXTEK Inc. Todo o sistema é portátil e pode ser usado em campo.

As condições de medida foram: $28 \mathrm{kV}, 10 \mu \mathrm{A}$ com filtro de $\mathrm{Ag}$ de $50 \mu \mathrm{m}$ na saída do tubo de raios $\mathrm{X}$.; colimador de $\mathrm{Ag}$ de $3 \mathrm{~mm}$ de abertura na entrada do detector e 500s de tempo de irradiação. 
Curva de calibração para EDXRF

Foram irradiados padrões mono-elementares depositadosemfilmes depolicarbonato(MicroMatter Inc.) para determinação da curva de sensibilidade.

Com o propósito de validar a metodologia, foram medidos dois materiais de referencia certificados do NIST (National Institute of Standards and Technology), SRM1640 e SRM1643e, ambos preparados com o uso da metodologia de préconcentração.

\section{Procedimento de quantificação por EDXRF}

A relação entre a intensidade dos raios $\mathrm{X}$ característicos de um dado elemento e sua concentração na amostra é dada pela equação dos parâmetros fundamentais, equação (1) (BERTIN, 1975):

$$
I_{i}=S_{i} C_{i} A
$$

onde: $\mathrm{I}_{i}$ é a intensidade dos raios $\mathrm{X}$ característicos (cps), $C_{i}$ é a concentração $\left(\mu \mathrm{g} \cdot \mathrm{g}^{-1}\right), \mathrm{S}_{\mathrm{i}}$ a sensibilidade elementar $\left(\mathrm{cps} \cdot \mathrm{g}^{-1} \cdot \mathrm{cm}^{2}\right)$ e A é o fator de absorção, considerado 1 para filmes finos.

O limite dedetecção foi determinado utilizando a equação (2) (CURIE, 1968):

$$
D L=\frac{3 \sqrt{B g}}{S t}
$$

onde: $\mathrm{Bg}$ são as contagens no fundo do espectro e $\mathrm{t}$ o tempo de medida.

\section{Resultados}

As curvas de sensibilidade estão nas Figuras (2) e (3). Na Tabela 1 está a concentração dos materiais de referencia certificados, com $95 \%$ de confiabilidade.

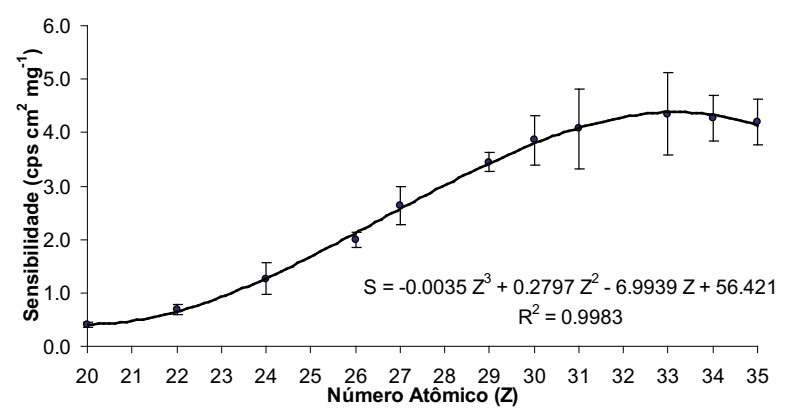

Figura 2. Curva de sensibilidade elementar para os raios $X$ característicos $\mathrm{K} \alpha$, utilizando os padrões MicroMatter.

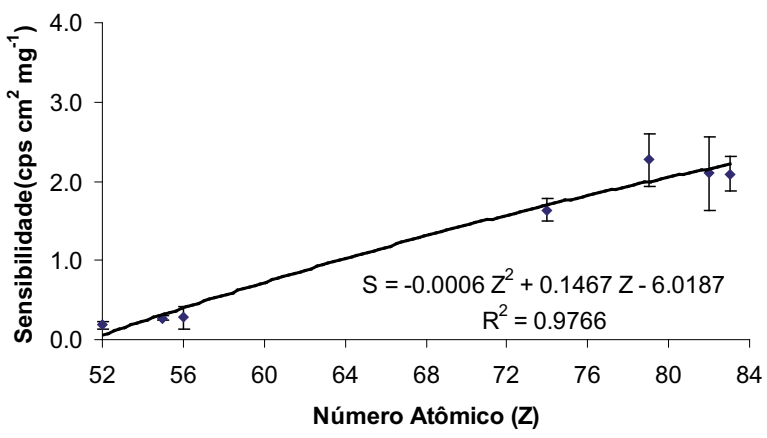

Figura 3. Curvas de sensibilidade elementar para os raios $\mathrm{X}$ característicos L $\alpha$, utilizando os padrões MicroMatter.

$\mathrm{Na}$ Tabela (2) estão apresentados os valores obtidos com a metodologia de EDXRF. Foi possível quantificar $\mathrm{Ca}$, Ti, $\mathrm{Mn}$, Fé, $\mathrm{Ni}, \mathrm{Cu}$ e Zn nas amostras. Para as amostras do Ribeirão Capivara, Córrego Água Fresca, Córrego Rubi e ribeirão Cambe, foi percebida uma pequena contaminação por Zn na coleta do inverno 2007, visto que o limite estabelecido pela Comissão Nacional do Meio Ambiente(CONAMA) (BRASIL, 2005) para águas classe 1 é $0,18 \mathrm{mg} \mathrm{L}^{-1}$. As concentrações para $\mathrm{Mn}$ e Ni ficaram abaixo do nível máximo permitido de $0,1 \mathrm{mg} \mathrm{L}^{-1}$ respectivamente 
Tabela 1. Resultados dos materiais de referencia certificados usando equipamento portátil de EDXRF. Valores com $95 \%$ de confiabilidade, em $\mathrm{mg} \mathrm{L}^{-1}$.

\begin{tabular}{ccccc}
\hline \multirow{2}{*}{ Elemento } & $\begin{array}{c}\text { Concentração } \\
\text { Certificada } \\
\text { SRM 1640 }\end{array}$ & $\begin{array}{c}\text { Concentração } \\
\text { Medida } \\
\text { SRM 1640 }\end{array}$ & $\begin{array}{c}\text { Concentração } \\
\text { Certificada } \\
\text { SRM 1643e }\end{array}$ & $\begin{array}{c}\text { Concentração } \\
\text { Medida } \\
\text { SRM 1643e }\end{array}$ \\
\hline $\mathrm{V}$ & $0.0130 \pm 0.0004$ & $0.006 \pm 0.002$ & $0.0216 \pm 0.0003$ & $0.012 \pm 0.004$ \\
$\mathrm{Fe}$ & $0.0343 \pm 0.0016$ & $0.047 \pm 0.003$ & $0.056 \pm 0.001$ & $0.110 \pm 0.008$ \\
$\mathrm{Co}$ & $0.0203 \pm 0.0003$ & $0.018 \pm 0.001$ & $0.0155 \pm 0.0002$ & $0.013 \pm 0.003$ \\
$\mathrm{Ni}$ & $0.0274 \pm 0.0008$ & $0.033 \pm 0.002$ & $0.0357 \pm 0.0004$ & $0.033 \pm 0.010$ \\
$\mathrm{Cu}$ & $0.0852 \pm 0.0012$ & $0.069 \pm 0.003$ & $0.0130 \pm 0.0002$ & $0.011 \pm 0.004$ \\
$\mathrm{Zn}$ & $0.0532 \pm 0.0011$ & $0.014 \pm 0.001$ & $0.045 \pm 0.001$ & $0.011 \pm 0.001$ \\
$\mathrm{Se}$ & - & - & $0.0068 \pm 0.0001$ & $0.006 \pm 0.001$ \\
$\mathrm{~Pb}$ & $0.0279 \pm 0.0001$ & $0.038 \pm 0.012$ & $0.0112 \pm 0.0001$ & $0.027 \pm 0.016$ \\
\hline
\end{tabular}

Tabela 2. Valores de concentração total e desvio padrão, em $\mathrm{mg} \mathrm{L}^{-1}$, para as amostras do Lago Igapó e seus afluentes.

\section{LAGO IGAPÓ I}

\begin{tabular}{cccl}
\hline Elemento & mar/06 & jun/06 & mai/07 \\
\hline $\mathrm{Ca}$ & $<0,009$ & $0,04 \pm 0,01$ & $0,04 \pm 0,01$ \\
$\mathrm{Ti}$ & $0,029 \pm 0,003$ & $0,12 \pm 0,01$ & $0,11 \pm 0,01$ \\
$\mathrm{~V}$ & $<0,004$ & $<0,006$ & $<0,004$ \\
$\mathrm{Cr}$ & $<0,003$ & $<0,004$ & $<0,003$ \\
$\mathrm{Mn}$ & $<0,002$ & 0,052 & $<0,002$ \\
$\mathrm{Fe}$ & $0,32 \pm 0,04$ & $0,82 \pm 0,04$ & $0,64 \pm 0,02$ \\
$\mathrm{Co}$ & $<0,001$ & $<0,001$ & $<0,001$ \\
$\mathrm{Ni}$ & 0,010 & $<0,001$ & $<0,001$ \\
$\mathrm{Cu}$ & $<0,001$ & $<0,001$ & $0,003 \pm 0,001$ \\
$\mathrm{Zn}$ & $<0,001$ & $0,003 \pm 0,001$ & $0,07 \pm 0,01$ \\
$\mathrm{As}$ & $<0,001$ & $<0,001$ & $<0,001$ \\
$\mathrm{Se}$ & $<0,001$ & $<0,001$ & $<0,001$ \\
$\mathrm{Hg}$ & $<0,001$ & $<0,002$ & $<0,002$ \\
$\mathrm{~Pb}$ & $<0,003$ & $<0,003$ & $<0,002$ \\
\hline
\end{tabular}

\section{LAGO IGAPÓ II}

\begin{tabular}{cccl}
\hline Elemento & mar/06 & jun $/ 06$ & mai/07 \\
\hline $\mathrm{Ca}$ & $<0,009$ & $0,032 \pm 0,005$ & $<0,010$ \\
$\mathrm{Ti}$ & $0,014 \pm 0,003$ & $0,051 \pm 0,007$ & $0,07 \pm 0,04$ \\
$\mathrm{~V}$ & $<0,004$ & $<0,005$ & $<0,005$ \\
$\mathrm{Cr}$ & $<0,003$ & $<0,003$ & $<0,003$ \\
$\mathrm{Mn}$ & $0,029 \pm 0,002$ & $0,043 \pm 0,003$ & $0,012 \pm 0,002$ \\
$\mathrm{Fe}$ & $0,25 \pm 0,06$ & $0,60 \pm 0,04$ & $0,56 \pm 0,11$ \\
$\mathrm{Co}$ & $<0,001$ & $<0,001$ & $<0,001$ \\
$\mathrm{Ni}$ & $0,012 \pm 0,001$ & $<0,001$ & $<0,001$ \\
$\mathrm{Cu}$ & $<0,001$ & $<0,001$ & $0,003 \pm 0,001$ \\
$\mathrm{Zn}$ & $<0,001$ & $<0,001$ & $0,077 \pm 0,002$
\end{tabular}

$\begin{array}{llll}\mathrm{As} & <0,001 & <0,001 & <0,001 \\ \mathrm{Se} & <0,001 & <0,001 & <0,001 \\ \mathrm{Hg} & <0,001 & <0,002 & <0,002 \\ \mathrm{~Pb} & <0,003 & <0,003 & <0,002\end{array}$

\section{LAGO IGAPÓ III}

\begin{tabular}{cccl}
\hline Elemento & mar/06 & jun/06 & mai/07 \\
\hline $\mathrm{Ca}$ & $<0,009$ & $<0,010$ & $0,02 \pm 0,01$ \\
$\mathrm{Ti}$ & $0,023 \pm 0,004$ & $0,033 \pm 0,004$ & $0,10 \pm 0,01$ \\
$\mathrm{~V}$ & $<0,003$ & $<0,004$ & $<0,005$ \\
$\mathrm{Cr}$ & $<0,002$ & $<0,003$ & $<0,004$ \\
$\mathrm{Mn}$ & $0,039 \pm 0,003$ & $0,008 \pm 0,001$ & $0,016 \pm 0,002$ \\
$\mathrm{Fe}$ & $0,37 \pm 0,03$ & $0,57 \pm 0,04$ & $0,92 \pm 0,09$ \\
$\mathrm{Co}$ & $<0,001$ & $<0,001$ & $<0,001$ \\
$\mathrm{Ni}$ & $0,011 \pm 0,003$ & $<0,001$ & $<0,001$ \\
$\mathrm{Cu}$ & $<0,001$ & $<0,001$ & $0,005 \pm 0,001$ \\
$\mathrm{Zn}$ & $<0,001$ & $<0,001$ & $0,102 \pm 0,004$ \\
$\mathrm{As}$ & $<0,001$ & $<0,001$ & $<0,001$ \\
$\mathrm{Se}$ & $<0,001$ & $<0,001$ & $<0,001$ \\
$\mathrm{Hg}$ & $<0,001$ & $<0,002$ & $<0,002$ \\
$\mathrm{~Pb}$ & $<0,002$ & $<0,002$ & $<0,002$ \\
\hline
\end{tabular}

\section{LAGO IGAPÓ IV}

\begin{tabular}{cccl}
\hline Elemento & mar $/ 06$ & jun $/ 06$ & mai $/ 07$ \\
\hline $\mathrm{Ca}$ & $<0,009$ & $<0,009$ & $<0,010$ \\
$\mathrm{Ti}$ & $0,027 \pm 0,015$ & $0,023 \pm 0,002$ & $0,024 \pm 0,005$ \\
$\mathrm{~V}$ & $<0,003$ & $<0,004$ & $<0,004$ \\
$\mathrm{Cr}$ & $<0,002$ & $<0,003$ & $<0,003$
\end{tabular}




\begin{tabular}{cccl}
$\mathrm{Mn}$ & $<0,001$ & $<0,002$ & $0,005 \pm 0,001$ \\
$\mathrm{Fe}$ & $0,66 \pm 0,14$ & $0,65 \pm 0,12$ & $0,62 \pm 0,04$ \\
$\mathrm{Co}$ & $<0,001$ & $<0,001$ & $<0,001$ \\
$\mathrm{Ni}$ & $0,010 \pm 0,003$ & $<0,001$ & $<0,001$ \\
$\mathrm{Cu}$ & $<0,001$ & $<0,001$ & $0,009 \pm 0,001$ \\
$\mathrm{Zn}$ & $<0,001$ & $<0,001$ & $0,14 \pm 0,01$ \\
$\mathrm{As}$ & $<0,001$ & $<0,001$ & $<0,001$ \\
$\mathrm{Se}$ & $<0,001$ & $<0,001$ & $<0,001$ \\
$\mathrm{Hg}$ & $<0,001$ & $<0,002$ & $<0,002$ \\
$\mathrm{~Pb}$ & $<0,002$ & $<0,002$ & $<0,002$ \\
\hline & & &
\end{tabular}

\section{CÓRREGO CAPIVARA}

\begin{tabular}{cccl}
\hline Elemento & mar/06 & jun/06 & mai/07 \\
\hline $\mathrm{Ca}$ & $<0,011$ & $0,04 \pm 0,01$ & $<0,012$ \\
$\mathrm{Ti}$ & $0,043 \pm 0,001$ & $0,14 \pm 0,02$ & $0,11 \pm 0,04$ \\
$\mathrm{~V}$ & $<0,002$ & $<0,006$ & $<0,004$ \\
$\mathrm{Cr}$ & $<0,001$ & $<0,004$ & $<0,003$ \\
$\mathrm{Mn}$ & $<0,001$ & $0,044 \pm 0,003$ & $<0,002$ \\
$\mathrm{Fe}$ & $0,28 \pm 0,02$ & $0,89 \pm 0,04$ & $0,71 \pm 0,10$ \\
$\mathrm{Co}$ & $<0,001$ & $<0,001$ & $<0,001$ \\
$\mathrm{Ni}$ & $0,009 \pm 0,005$ & $<0,001$ & $<0,001$ \\
$\mathrm{Cu}$ & $<0,001$ & $<0,001$ & $0,004 \pm 0,001$ \\
$\mathrm{Zn}$ & $<0,001$ & $<0,001$ & $0,19 \pm 0,02$ \\
$\mathrm{As}$ & $<0,001$ & $<0,001$ & $<0,001$ \\
$\mathrm{Se}$ & $<0,001$ & $<0,001$ & $<0,001$ \\
$\mathrm{Hg}$ & $<0,001$ & $<0,002$ & $<0,002$ \\
$\mathrm{~Pb}$ & $<0,003$ & $<0,003$ & $<0,002$ \\
\hline
\end{tabular}

\section{CÓRREGO LEME}

\begin{tabular}{cccl}
\hline Elemento & mar/06 & jun/06 & mai/07 \\
\hline $\mathrm{Ca}$ & $<0,009$ & $<0,010$ & $0,02 \pm 0,01$ \\
$\mathrm{Ti}$ & $0,015 \pm 0,003$ & $<0,005$ & $0,04 \pm 0,01$ \\
$\mathrm{~V}$ & $<0,001$ & $<0,004$ & $<0,003$ \\
$\mathrm{Cr}$ & $<0,001$ & $<0,002$ & $<0,005$ \\
$\mathrm{Mn}$ & $<0,001$ & $<0,003$ & $<0,002$ \\
$\mathrm{Fe}$ & $0,18 \pm 0,02$ & $0,12 \pm 0,01$ & $0,36 \pm 0,06$ \\
$\mathrm{Co}$ & $<0,001$ & $<0,001$ & $<0,001$ \\
$\mathrm{Ni}$ & $0,005 \pm 0,003$ & $<0,001$ & $<0,001$ \\
$\mathrm{Cu}$ & $<0,001$ & $<0,001$ & $0,003 \pm 0,001$ \\
$\mathrm{Zn}$ & $<0,001$ & $<0,001$ & $0,055 \pm 0,005$ \\
$\mathrm{As}$ & $<0,001$ & $<0,001$ & $<0,001$ \\
$\mathrm{Se}$ & $<0,001$ & $<0,001$ & $<0,001$ \\
$\mathrm{Hg}$ & $<0,001$ & $<0,002$ & $<0,002$ \\
$\mathrm{~Pb}$ & $<0,003$ & $<0,003$ & $<0,002$ \\
\hline
\end{tabular}

CÓRREGO ÁGUA FRESCA

\begin{tabular}{cccl}
\hline Elemento & mar/06 & jun $/ 06$ & mai/07 \\
\hline $\mathrm{Ca}$ & $<0,010$ & $0,021 \pm 0,002$ & $0,017 \pm 0,009$ \\
$\mathrm{Ti}$ & $0,006 \pm 0,002$ & $0,016 \pm 0,004$ & $0,07 \pm 0,02$ \\
$\mathrm{~V}$ & $<0,001$ & $<0,004$ & $<0,005$ \\
$\mathrm{Cr}$ & $<0,001$ & $<0,003$ & $<0,003$ \\
$\mathrm{Mn}$ & $0,005 \pm 0,002$ & $0,042 \pm 0,015$ & $0,010 \pm 0,002$ \\
$\mathrm{Fe}$ & $0,17 \pm 0,05$ & $0,31 \pm 0,04$ & $0,63 \pm 0,09$ \\
$\mathrm{Co}$ & $<0,001$ & $<0,001$ & $<0,001$ \\
$\mathrm{Ni}$ & $0,006 \pm 0,003$ & $<0,001$ & $<0,001$ \\
$\mathrm{Cu}$ & $<0,001$ & $<0,001$ & $0,004 \pm 0,001$ \\
$\mathrm{Zn}$ & $<0,001$ & $<0,001$ & $0,30 \pm 0,02$ \\
$\mathrm{As}$ & $<0,001$ & $<0,001$ & $<0,001$ \\
$\mathrm{Se}$ & $<0,001$ & $<0,001$ & $<0,001$ \\
$\mathrm{Hg}$ & $<0,001$ & $<0,002$ & $<0,002$ \\
$\mathrm{~Pb}$ & $<0,003$ & $<0,003$ & $<0,003$ \\
\hline
\end{tabular}

\section{CÓRREGO RUBI}

\begin{tabular}{cccl}
\hline Elemento & mar/06 & jun/06 & mai/07 \\
\hline $\mathrm{Ca}$ & $<0,009$ & $<0,009$ & $<0,009$ \\
$\mathrm{Ti}$ & 0,000 & $0,014 \pm 0,005$ & $0,033 \pm 0,003$ \\
$\mathrm{~V}$ & 0,000 & $<0,004$ & $<0,004$ \\
$\mathrm{Cr}$ & $<0,02$ & $<0,003$ & $<0,003$ \\
$\mathrm{Mn}$ & 0,000 & $<0,002$ & $<0,002$ \\
$\mathrm{Fe}$ & $0,25 \pm 0,01$ & $0,18 \pm 0,03$ & $0,24 \pm 0,01$ \\
$\mathrm{Co}$ & $<0,001$ & $<0,001$ & $<0,001$ \\
$\mathrm{Ni}$ & $<0,001$ & $<0,001$ & $<0,001$ \\
$\mathrm{Cu}$ & $<0,001$ & $<0,001$ & $0,004 \pm 0,001$ \\
$\mathrm{Zn}$ & $<0,001$ & $<0,001$ & $0,295 \pm 0,010$ \\
$\mathrm{As}$ & $<0,001$ & $<0,001$ & $<0,001$ \\
$\mathrm{Se}$ & $<0,001$ & $<0,001$ & $<0,001$ \\
$\mathrm{Hg}$ & $<0,0001$ & $<0,002$ & $<0,002$ \\
$\mathrm{~Pb}$ & $<0,002$ & $<0,003$ & $<0,002$ \\
\hline
\end{tabular}

CÓRREGO BARORÉ

\begin{tabular}{cccl}
\hline Elemento & mar/06 & jun/06 & mai/07 \\
\hline $\mathrm{Ca}$ & $<0,010$ & $<0,010$ & $<0,009$ \\
$\mathrm{Ti}$ & $0,02 \pm 0,01$ & $<0,004$ & $<0,004$ \\
$\mathrm{~V}$ & $<0,001$ & $<0,003$ & $<0,003$ \\
$\mathrm{Cr}$ & $<0,001$ & $<0,002$ & $<0,002$ \\
$\mathrm{Mn}$ & $<0,001$ & $<0,002$ & $<0,002$ \\
$\mathrm{Fe}$ & $0,46 \pm 0,06$ & $0,33 \pm 0,02$ & $0,35 \pm 0,02$ \\
$\mathrm{Co}$ & $<0,001$ & $<0,001$ & $<0,001$ \\
$\mathrm{Ni}$ & $<0,001$ & $<0,001$ & $<0,001$ \\
$\mathrm{Cu}$ & $<0,001$ & $<0,001$ & $0,004 \pm 0,001$ \\
$\mathrm{Zn}$ & $<0,001$ & $<0,001$ & $0,17 \pm 0,01$ \\
$\mathrm{As}$ & $<0,001$ & $<0,001$ & $<0,001$
\end{tabular}




\begin{tabular}{clll}
$\mathrm{Se}$ & $<0,001$ & $<0,001$ & $<0,001$ \\
$\mathrm{Hg}$ & $<0,001$ & $<0,002$ & $<0,002$ \\
$\mathrm{~Pb}$ & $<0,002$ & $<0,002$ & $<0,002$ \\
\hline
\end{tabular}

\section{RIBEIRÃO CAMBÉ}

\begin{tabular}{cccl}
\hline Elemento & mar/06 & jun/06 & mai/07 \\
\hline $\mathrm{Ca}$ & 0,000 & $<0,010$ & $<0,019$ \\
$\mathrm{Ti}$ & $0,006 \pm 0,001$ & $0,023 \pm 0,006$ & $0,29 \pm 0,09$ \\
$\mathrm{~V}$ & $<0,001$ & $<0,004$ & $<0,008$ \\
$\mathrm{Cr}$ & $<0,001$ & $<0,003$ & $<0,004$ \\
$\mathrm{Mn}$ & $<0,001$ & $<0,002$ & $<0,002$ \\
$\mathrm{Fe}$ & $0,27 \pm 0,02$ & $0,72 \pm 0,04$ & $2,5 \pm 0,3$ \\
$\mathrm{Co}$ & $<0,001$ & $<0,001$ & $<0,002$ \\
$\mathrm{Ni}$ & $0,005 \pm 0,003$ & $<0,001$ & $<0,001$ \\
$\mathrm{Cu}$ & $<0,001$ & $<0,001$ & $0,006 \pm 0,001$ \\
$\mathrm{Zn}$ & $<0,001$ & $<0,001$ & $0,215 \pm 0,010$ \\
$\mathrm{As}$ & $<0,001$ & $<0,001$ & $<0,001$ \\
$\mathrm{Se}$ & $<0,001$ & $<0,001$ & $<0,001$ \\
$\mathrm{Hg}$ & $<0,001$ & $<0,002$ & $<0,002$ \\
$\mathrm{~Pb}$ & $<0,002$ & $<0,003$ & $<0,002$ \\
\hline
\end{tabular}

Para o $\mathrm{Fe}$ e o $\mathrm{Cu}$, cujos limites de concentração na legislação referem-se à fração dissolvida do metal, os valores são 0,3 e $0,009 \mathrm{mg} \mathrm{L}^{-1}$, respectivamente (BRASIL, 2005). Para o $\mathrm{Cu}$ não foi identificada contaminação em nenhuma das amostras. No caso do $\mathrm{Fe}$, a quantidade de metal dissolvido variou de $0,003 \pm 0,001$ a $0,590 \pm 0,027 \mathrm{mg} \mathrm{L}^{-1}$. Os pontos com possível contaminação podem ser identificados na Figura (4), por meio da qual pode ser inferida a dinâmica do ferro dissolvido nos pontos avaliados. O Ribeirão Cambé e o Córrego Baroré desembocam no Lago Igapó IV, que por sua vez abastece os demais lagos. Analisando a concentração de ferro dissolvido nos lagos, percebe-se que ela vai diminuindo do Lago Igapó IV até o Lago Igapó I, sugerindo que o ferro está sendo diluído no percurso.

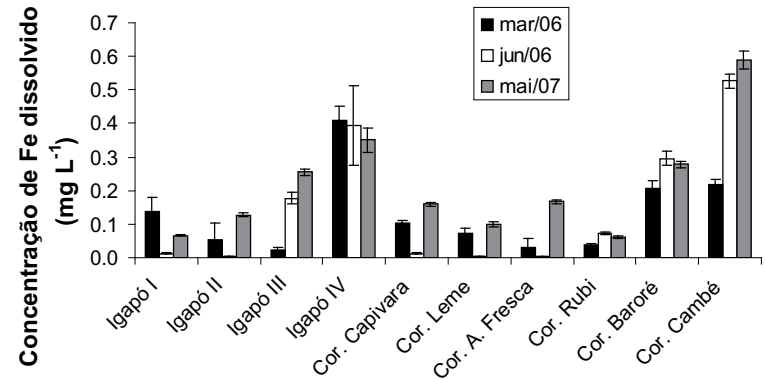

Figura 4. Valores de concentração para Fe dissolvido nas amostras do Lago Igapó medidas por EDXRF portátil. A linha tracejada indica o limite estabelecido pelo CONAMA para águas classe 1 .

A variação de concentração de $\mathrm{Ca}, \mathrm{Ti}, \mathrm{Mn}$ e $\mathrm{Fe}$ durante o período de monitoração pode ser visto nos gráficos da Figura (5). O titânio, encontrando somente na fração não dissolvida, é proveniente do solo contido no material particulado em suspensão. No Ribeirão Cambe, na coleta de mai/07, foi percebida uma elevada concentração de Fe e Ti no material particulado em suspensão, o que refletiu na concentração total, e, para o caso do $\mathrm{Fe}$, a fração dissolvida é semelhante à coleta de jun/06 (ver Figura 4).

O Instituto Ambiental do Paraná avaliou a presença de $\mathrm{Cr}, \mathrm{Fe}, \mathrm{Cd}, \mathrm{Hg}$ e $\mathrm{Pb}$ nas amostras, utilizando a técnica de Espectrometria de absorção atômica com chama (FAAS). Nessa análise, foram encontrados apenas valores para Fe acima do limite de detecção do sistema utilizado. Sendo assim, as concentrações de Fe puderam ser comparadas entre as duas técnicas. A Figura (6) traz esta comparação, mostrando que existe boa correlação entre os resultados. 

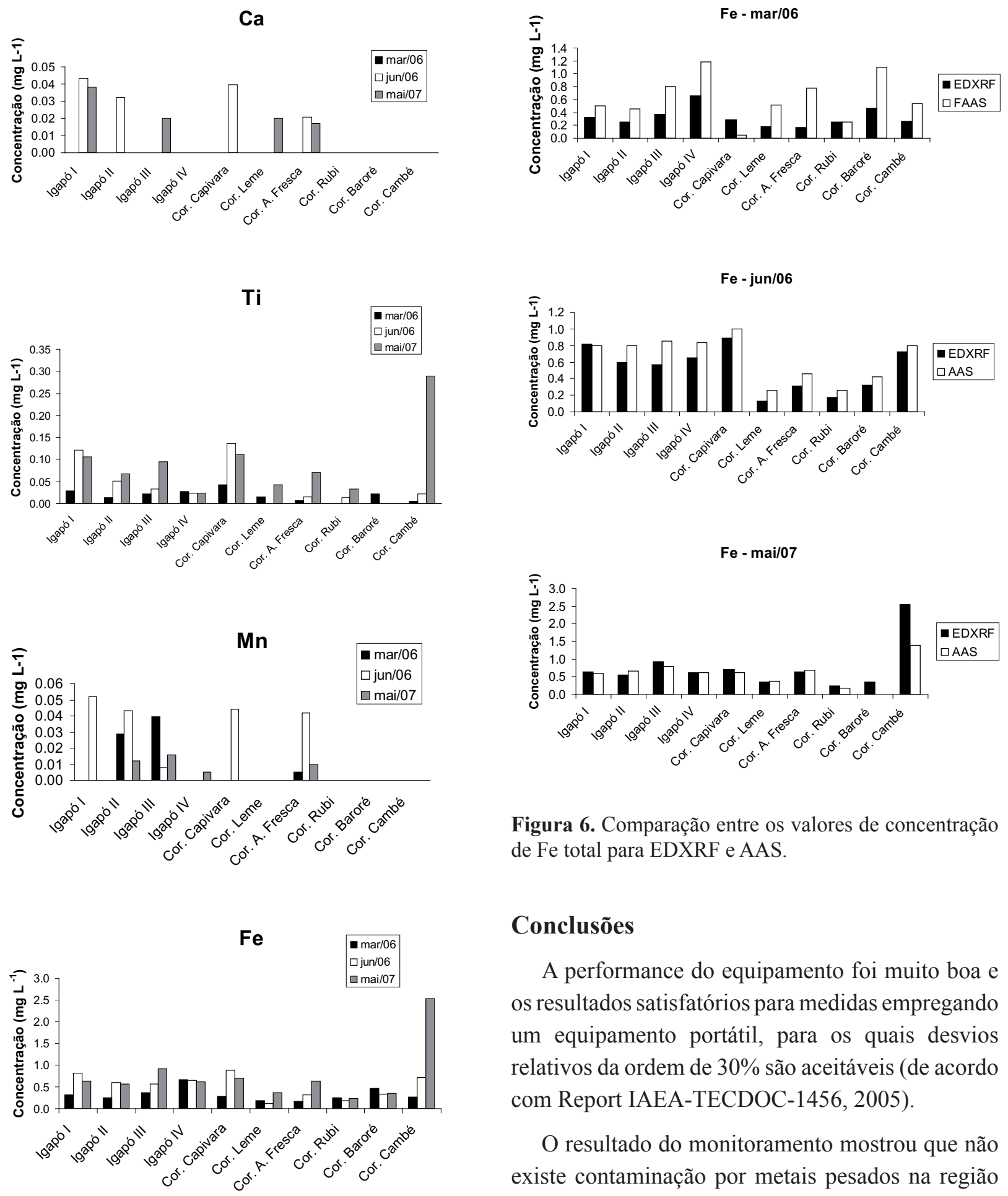

Figura 5. Valores de concentração total, em $\mathrm{mg} \mathrm{L}^{-1}$, do monitoramento de $\mathrm{Ca}, \mathrm{Ti}$, Mn e Fe.

Figura 6. Comparação entre os valores de concentração de Fe total para EDXRF e AAS.

\section{Conclusões}

A performance do equipamento foi muito boa e os resultados satisfatórios para medidas empregando um equipamento portátil, para os quais desvios relativos da ordem de 30\% são aceitáveis (de acordo com Report IAEA-TECDOC-1456, 2005).

O resultado do monitoramento mostrou que não existe contaminação por metais pesados na região durante o período amostrado. A presença de $\mathrm{Ca}$, Ti, $\mathrm{Mn}, \mathrm{Fe}($ dissolvido), Ni, $\mathrm{Cu}$ e $\mathrm{Zn}$ foi identificada somente por EDXRF, devido à sua facilidade de análise multi-elementar, e não foi identificada por 
AAS. A metodologia de pré-concentração permitiu a quantificação da fração dissolvida e não dissolvida dos metais sem a necessidade de procedimentos exaustivos.

Os resultados das duas metodologias geraram conclusões mais minuciosassobre as condições da água nos pontos analisados, complementado a análise de rotina realizada frequentemente.

A EDXRF portátil mostrou-se uma ferramenta muito útil para análises ambientais, por ser uma técnica com alta velocidade analítica, eficiente e conveniente, com potencial para substituir métodos laboratoriais demorados e com custo mais elevado. A metodologia pode ser utilizada no mapeamento de regiões para identificação de pontos com maior contaminação.

\section{Agradecimentos}

Ao Instituto Ambiental do Paraná, especialmente à Leda Neiva Dias e Luis Zaransky pela colaboração na amostragem e discussão sobre a metodologia e resultados de AAS. Também somos gratos à Fundação Araucária de Apoio ao Desenvolvimento Científico e Tecnológico do Paraná (009/20055402) e ao Conselho Nacional de Desenvolvimento Tecnológico, CNPq (470662/2004-2), pelo apoio financeiro.

\section{Referências}

AMPTEK Inc., Operating manual - XR-100CR x-ray detector system and PX2CR power supply/shaper. 1998.

BERTIN, E. P. Principles and practice of X-ray spectrometric analysis. London: Plenum Press, 1975. $1079 \mathrm{p}$.

BRASIL. Ministério do Meio Ambiente, Conselho Nacional do Meio Ambiente. Resolução $n^{\circ} 375$, de 17 de março de 2005. Dispõe sobre a classificação dos corpos de água e diretrizes ambientais para o seu enquadramento, bem como estabelece as condições e padrões de lançamento de efluentes, e dá outras providências. Brasília, 2005.

CESAREO, R. X-ray physics: Interaction with matter, production, detection. La Revista des Nuovo Cimento della Società Italiana di Fisica, Editrice Compositori, Bologna, v. 23, n.76, p. 1-231, 2000.

CESAREO, R.; CASTEllanO, A.; CUEVAS, A. M. Energy dispersive $\mathrm{x}$-ray fluorescence analysis of thin and intermediate environmental samples. X-ray Spectrometry, London, v.27, n. 4, p. 257-264, 1998.

CURIE, L. A. Limits for quantitative detection and quantitative determination. Analytical Chemistry, Washington, v. 40, n. 3, p. 586-593, 1968.

HASSAN, N. M.; RASMUSSEN, P. E.; DABEKZLOTRZYNSKA, E.; CELO, V.; CHEN, H. Analysis of environmental samples using microwave assisted acid digestion and Inductively Coupled Plasma Mass Spectrometry - Maximizing total element recoveries. Water Air Soil Pollution, Dordrecht, v. 178, n. 1/4, p. 323-334, 2007.

HOU, X.; JONES, B. T. Field instrumentation in atomic spectroscopy. Microchemical Journal, New York, v. 66, n. $1 / 3$, p. $115-145,2000$.

IAEA-TECDOC-1456. In situ applications of X-ray fluorescence techniques. Viena, 2005.

IVANOVA, J. U.; DJINGOVA, R.; KULEFF, I. Determination of some heavy and toxic elements in plants and soils with ED-XRF using 241Am excitation sources. Journal of Radioanalytical and Nuclear Chemistry, Lousanne, v. 28, n. 1/2, p. 29-32, 1998.

MELQUIADES, F. L.; APPOLONI, C. R. Application of XRF and field portable XRF for environmental analysis. Journal of Radioanalytical and Nuclear Chemistry,Lousanne, v. 262, n. 2, p. 533-541, 2004.

MELQUIADES, F. L; PARREIRA, P. S.; YABE, M. J.; CORAZZA, M. Z.; FUNFAS, R. Factorial design for Fe, $\mathrm{Cu}, \mathrm{Zn}$, Se and $\mathrm{Pb}$ preconcentration optimization with APDC and analysis with a portable X-Ray fluorescence system, Talanta, London,v. 73, n. 1, p. 121-126, 2007.

MOXTEK Inc. Operating manual: miniature bullet X ray tube. 2003 .

VAN GRIEKEN, R.; ARAUJO, F.; ROJAS, C.; VENY, P. $X R F$ and PIXE applications in life sciences. Capri (Italy), 29-30 June 1989. World Scientific, Singapore, 1990. p. 79. 
